\title{
Detailed Case Study about Smog Eating Tile
}

\author{
Amit Dhundalwar ${ }^{1}$, Tauheed Naseeb Alam Ansari ${ }^{2}$, Razaul Mustufa Khan ${ }^{3}$, \\ Sopan Sawant ${ }^{4}$, Vineet Wasnik ${ }^{5}$, Prof. Dr. P. S. Lanjewar ${ }^{6}$, Prof. Ashish Moon ${ }^{7}$ \\ 1,2,3,4,5 Students ${ }^{6,7}$ Professor \\ Smt. Radhikatai Pandav College of Engineering, Nagpur, India, 440034
}

Received on: 25 May,2021

Revised on: 28 June,2021

Published on: 30 June,2021

\begin{abstract}
Our Project is about to study the ill effects of smog and air pollution and control it by using smog eating tiles. Our aim is to study the economy of this construction and contribute our part in 'SWACHA BHARAT ABHIYAN'. The rapid growth in pollution is due to excessive use of vehicles. Previous and growing Industries such as steel industries, thermal electric power plants, etc. These tiles are normal roofing tiles which are installed on the roof. The only thing which differs is titanium dioxide on it. The coating of titanium dioxide (single coat) is applied on the tiles which can be done in the form of paint. Titanium dioxide is available in the form of powder it is mixed with water and prepared in the form of paint. It is applied on the roofing tiles with painting tools. Cost of these tiles is just $25 \%$ more than the normal roofing tiles.
\end{abstract}

Keywords- Smog Eating Tiles

\section{I - INTRODUCTION}

T here is a growing account of air pollution and the outbreak of smog every year in one of the parts of the world. Huge amount of pollutant gases is emitted by burning of fuels through the consumptive or productive activities. The air pollution has been one of the biggest concerns of the environmental protection agencies all through the world owing to the hazards it causes to human health, monuments and ecology. An average human being consumes about 10,000 to 20,000 liters of air every day; there is an intake of 7-14 liters of air by us in a minute. This certainly points out the criticality of clean air. It is said that more than the external effects that a smog produces, like choking hazards etc., it reduces the immune system, so more deaths are due to the weakening immune system of human beings. The smog is as simple as that, smog is a combination of smoke and fog, but what lies within smog? Here is a deep insight on the same.

The research and the project revolve around one basic concept of not letting the nitrogen oxide interact with sunlight. This could be achieved by introducing a compound which helps in the absorption of nitrogen oxide. The solution which was developed by the university students involves coating the roof tiles of residential building by titanium dioxide. They call it as "smog eating roof tiles". In the course of a year, 2000 square feet of SMOG EATING TILE reduces the same amount of nitrogen oxide that produced by one car driving up to 10,800 miles, thus smog eating tile is used to solve issues.

\section{II - LITERATURE REVIEW}

1. Mark Garger, Evan Marohn -With pollution everincreasing in a rapidly developing world, environmental protection will rely on new innovations to remove pollutants. One such innovation is titanium dioxide- 


\section{International Journal of Innovations in Engineering and Science, www.ijies.net}

infused concrete and cement, which can remove smog from the air when exposed to ultraviolet light. To clean the air of smog, the nitrogen oxides present must be transformed into different, less harmful substances such as nitrate ions. The nitrate ions formed during the reaction then create nitric acid, which is reacted with grout to create a harmless neutral salt. The salt is then washed out by precipitation. While this reaction can occur naturally, it does so at a slow rate. The process is sped up by the presence of titanium dioxide, which acts as a photocatalyst, meaning it can speed up a reaction when light is also present. The technology has already been implemented into various structures and has been subject to performance tests. The titanium oxide-infused concrete has shown to have greater flexural and compressive strength than normal concrete, giving a structural as well as environmental advantage. Furthermore, the titanium oxide concrete proves to effectively remove nitrogen oxides as well as common volatile organic compounds (VOCs) such as benzene, toluene, and ethylbenzene. This material has been integrated into materials ranging from roofing tiles to main structural components of buildings, and proves to be a sustainable solution.

2. Laurent Barcelo- EPA lists Nitrogen Oxides (NO and $\mathrm{NO} 2$, together referred to as "NOx") as one of the six common air pollutants. NOx is present in emissions from transportation (cars, trucks, etc.) and also in power plant and other combustion process emissions. The more toxic form, NO2, is recognized to have extensive direct effects on human health (such as asthma and acute respiratory diseases) and also on many other organisms, especially aquatic life forms (due to eutrophication from atmospheric deposition). NOx additionally affects the environment by promoting acid rain and the formation of ground-level ozone, which causes smog and is harmful to sensitive plant life and ecosystems. The technology used in "smog-eating" (or "de-polluting") concrete helps mitigate the effect of atmospheric NO2. Contrary to other solutions, this technology does not rely on photocatalysis and therefore can function perfectly well in the dark. So it is especially suitable for use in confined areas prone to very high NO2 concentrations, such as tunnels or parking garages. Research shows that all concretes have some tendency to absorbNO2. However, this tendency depends on the concrete mix design, and it is also reduced significantly over time by carbonation. The use of small additions of activated carbon can greatly enhance the NO2 absorption properties of many classes of concrete and also reduces the negative influence of carbonation the process. The use of "smog-eating concrete" may provide a way to improve our cities. By reducing ambient NOx concentrations, it will have a benefice impact on human health as well as on animal and plant life.

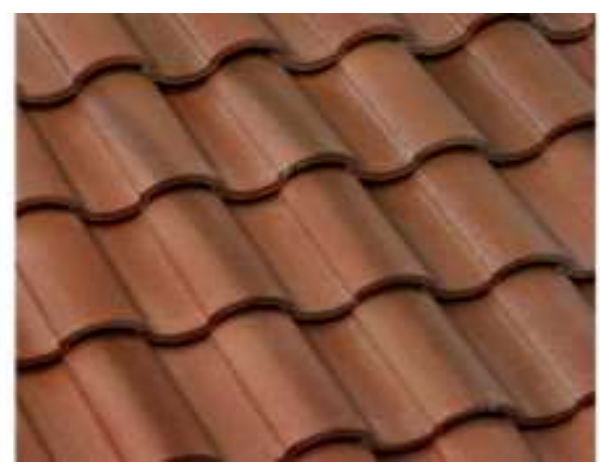

Fig. 1-fig Smog Eating Tiles

3. Gabe Klein -The Pilsen Sustainable Streetscape spans a 2-mile stretch of West Cermak Road in the Pilsen neighbourhood of Chicago, including the intersection at Blue Island. The project was completed in Spring 2013 and marks the first time that photocatalytic (smogeating) concrete has been used in the United States, earning the streetscape its title as the "Greenest Street in America." While the Pilsen neighbourhood is experiencing a transition to a more mixed-use neighbourhood, industrial areas nearby make West Cermak a common truck route, and an excellent opportunity to utilize smog-eating concrete. The streetscape also includes many other sustainable features, such as permeable pavement and bioswales for stormwater management, solar- and wind-powered streetlights, drought-resistant plants for landscaping, and bike lanes to encourage non-polluting modes of transport. In addition, $23 \%$ of construction materials used for the project contained recycled material and $60 \%$ of construction waste produced was recycled.

4. Abhijeet Shukla- Pollution controlling and smogeating properties induced in a self-cleaning cementitious material were identified recently. Initially meant as a self-cleaning material, it was discovered that this material had the ability to reduce certain pollutants from surrounding air, and this reduction in the concentration of the pollutants depends upon the surrounding conditions and the amount of exposed area. These properties induced in cementitious material were mainly due to the photocatalytic action of $\mathrm{TiO} 2$, which under 


\section{International Journal of Innovations in Engineering and Science, www.ijies.net}

the influence of sunlight acts as a catalyst and triggers chemical reaction due to that decomposition of dirt particles take place on the concrete's surface, also it reacts with certain pollutants that contribute to smog, breaking it under the presence of sunlight and accelerating their natural oxidation process. It is found that it is very effective against the Nitrogen oxides (NOx), Sulphur oxides (SOx) and many other harmful pollutants, which are mainly released due to the burning of fossil fuels and is one of the major constituents of smog. It decomposes NOx effectively up to a great extent in the surrounding environment and to a height of about 2-3 meters above the surface. In simple words, this material showing photocatalytic property due to $\mathrm{TiO} 2$, when irradiated with sunlight accelerates the decomposition process of pollutants and dirt clearing up the smog and ultimately cleaning the environment and that is the reason why the term self-cleaning and smogeating is given to it. This material has already found relevant applications and is being used in a variety of projects such as self-cleaning walls and in the reduction of urban pollutants and serves multiple purposes. Furthermore, the main focus of this paper is to explain the mechanisms involved and to understand the merits and demerits of using this cementitious material in a better way and also it aims at finding out ways in which it can contribute more to this new environment friendly civil engineering world.

\section{III - WORKING}

The first and only product of its kind in the U.S. roofing industry, smog-eating tile integrates titanium dioxide (TiO2) into a micro mortar coating over the upper tile body. Safely embedded in the coating, titanium dioxide is found in many consumer and industrial products; and when exposed to sunlight, it speeds up the oxidation process of nitrogen oxide (NOx), a major component of smog that is found in high levels in major metropolitan regions where cars and freeways are prolific. Smogeating tile oxidizes harmful NOx molecules released from vehicles, helping to make air safer to breathe. The roof coating contains a photocatalyst, activated by daylight, which helps convert harmful nitrous oxides into calcium nitrates. When it rains, the calcium nitrates are then washed off the roof.

Some major health problems are directly attributable to the NOx formed during any burning process. Over one year, 2,000 sq. ft. of the tile can oxidize as much NOx as a car produces from being driven up to 10,800 miles. Thus, the tile's air cleansing process represents one real way that builders and homeowners alike can reduce smog and the occurrence of the health problems it causes. "Smog-Eating Tiles" improve air quality by neutralizing smog-forming nitrogen oxides released by most vehicles (read more about smog in our fact box).

The tiles are coated with titanium dioxide, a photocatalyst that can oxidize harmful air pollutants emitted in the burning of fossil fuels. When exposed to natural light, the titanium dioxide breaks down nitrogen oxides in the air and turns them into harmless calcium nitrate. The Boral's Energy Efficient Roof System can help you to save up to $22 \%$ in energy costs. The roof system helps keep your home warmer in winter and cooler in summer. Helps to reduce your heat and air conditioning bills. However, just like other high-tech productions its price is approximately $\$ 650$ more than a standard 2500sq.ft. tile roof.

\section{IV - CONCLUSION}

- Benefits outweigh the costs.

- Smog level reduction creates a healthier environment and inhabitants.

- Tiles reduce home heating and cooling costs.

- Recyclable tiles mitigate the amount of landfill waste.

- It has been observed that smog has been reduced to minimum level due to Titanium dioxide. Therefore, it is an eco-friendly advice to the industries that they should install this on their roof which are the larger producers of smog. This idea can be useful for our future and it should be implemented in India at various polluted cities such as DELHI, CHANDRAPUR, MUMBAI etc. To reduce or minimize number of air pollutants to achieve clean air to breath.

- This project is move towards our contribution for eco-friendly environment in metro cities subjected to high level of pollution due to industries and traffic.

- An on the conclusion of our project is to give contribution in 'SWACHHA BHARAT ABHIYAN' like the country in which we are living, not only its land should be cleaned but also its air should be purified with every polluting agent which endangers the environment and human health. 


\section{REFERENCES}

[1] Beeidens Anne Environmental friendly Concrete pavement blocks air purification in the centre of Antwerp, presented at the 8th international conference on concrete paving, NOV 6-8

[2] San fransisco, CA Chai-Mei Yu jimmy. Deactivation and regeneration of environmentally exposed titanium dioxide based products. Testing report prepared for environmental protection department HAKAR June 2006.

[3] Chusid Michael "Next Step: Self Cleaning Concrete! Photo catalysts can keep concrete clean and reduce air pollution." New Technology. Aug/Sept 2005.chusid Michael "words you should know: depollution, photo catalysis" precast solutions Magazine, Nov.2006http://www.precast .org/publications/solutions/2006_fall/feature_words. htmElegant Embellishments product information at http://www, elegantembellishments.net/eraser,Lance. "Titanium Dioxide; Environmental White Knights?" Environmental Health Perspectives. Vol 109, Number 4, April 2001.

[4] Maggots, T, Kitties J., Bartziz,j., Leva R Bellintani, A.., and Vasilakos, C. " Invwatigations of Tio2 Contaning Materials for decompositions of NOxln Environment Chambers". http://www.jrc.cec.eu.int/pce/documentation/eur_rep orts/Investigations_Tio2_UAQ2005.pdfMamaugh , Geoff Urban Grids /Respiratory Oasis. World Changing.Oct 10, 2006.

[5] "Pollution Eating Concrete". http://www.treehunger.com/files/2005/07/pollutiom_ eatin.phpPort of Lost Angeles website. http://www.portoflosangeles.orgPort of Los Angeles News Release http://www.portoflosangeles.org/Press/REL_CAAP Final.pdfProvoledo, Elisabetta "Architecture in Italy Goes Green”. International Herald Tribune.Nov.2006.Provoledo, Elisabetta. "Church on the Edge of Rome Offers a Solution to Smog”. The NewsTimes.Nov.2006.

[6] San Pedro Bay Ports Clean Air Action Plan Overview.http://www.portoflosangeles,org/DOC/RE PORT_Clean_Air_Overview_Englishpdf16|20 December 2006 Toto Newsletter. “TOTO's Work with Environmentally Friendly Photocatalyst Technology."No.36.Dec.2003. Wang, Linda. “A Common Whitener Helps to Clean Air'.Scientific America Feb.2002. 